\title{
Atomic Structure and Energetic Stability of the Bi-covered InAs(110) Surface
}

\author{
R. H. Miwa and E. K. Takahashi \\ Faculdade de Física, Universidade Federal de Uberlândia \\ Caixa Postal 593, 38400-902, Uberlândia, MG, Brazil
}

Received on 10 March, 2003

\begin{abstract}
We have performed $a b$ initio calculations of the atomic structure and energetic stability of the $\mathrm{Bi}$-covered InAs(110) surface. The calculations were performed within the density-functional theory, using normconserving fully separable $a b$ initio pseudopotentials. Two experimentally proposed structural models have been considered: $(1 \times 1)$-ECLS and $(1 \times 2)$. Our total energy calculations indicate that the formation of the $(1 \times 2)$ model is energetically more favourable than the $(1 \times 1)$ model by $41 \mathrm{meV} /(1 \times 2)$. The calculated equilibrium geometries, for the $\operatorname{In} \mathrm{As}(110)-\mathrm{Bi}(1 \times 1)$ and $-\mathrm{Bi}(1 \times 2)$ surfaces, show in general a good agreement with the experimental x-ray measurements carried out by Betti et al. [Phys. Rev. B, 59, 15760 (1999)].
\end{abstract}

\section{Introduction}

The atomic and electronic structures of two-dimensional semimetal adlayers on semiconductor surfaces have been subject of many experimental studies. It is well known that the $\mathrm{Sb}$ - and $\mathrm{Bi}$-covered $\mathrm{GaAs}(110)$, and $\mathrm{Sb}$-covered InAs(110) surfaces exhibit a $(1 \times 1)$ surface reconstruction [1-6] However, a very recent experimental work for the Bi-covered InAs(110) surface, using $x$-ray diffraction technique, identified an InAs missing row between two adjacent $\mathrm{Bi}-$ chains, forming a $(1 \times 2)$ surface reconstruction [7].

In this work we have studied the Bi-covered InAs(110) surface. Two different structural models have been considered: the $(1 \times 1)$ reconstructed epitaxial continued layer structure $($ ECLS) [8] and the $(1 \times 2)$ reconstructed missingrow model [7].

\section{Method of Calculation}

The calculations were performed in the framework of the density functional theory [9], within the local density approximation using the Ceperley-Alder correlation [10] as parameterized by Perdew and Zunger [11]. The electron-ion interaction was treated by using norm-conserving, ab initio, fully separable pseudopotentials [12]. The wave functions were expanded in a plane wave basis set with a kinetic energy cutoff of $16 \mathrm{Ry}$. The theoretical lattice constant of $5.98 \AA$ were obtained for InAs. To simulate the surfaces we used the repeated slab method [13], with a supercell containing eight atomic layers plus $\approx 11 \AA$ of vacuum region. A layer of fractionally charged hydrogen atoms was used to saturate the cation dangling bonds at the bottom layer of the slab. The electronic charge density was calculated using a set of 4 special k-points in the irreducible part of the surface Brillouin zone. The eight topmost layers were fully relaxed within a force convergence criterion of $12 \mathrm{meV} / \AA$.

\section{Results and Discussion}

Figure 1 exhibits the structural models and details of the equilibrium atomic geometry for the $\mathrm{Bi}$-covered $\operatorname{InAs}(110)$ surface: $(1 \times 1)$ ECLS [Figs. 1(a) and (b)] and the $(1 \times 2)$ missing-row model [Figs. 1(c) and (d)]. In the ECLS the Bi adatoms occupy the III-V sites in the (110) plane of the InAs adlayer, forming $\mathrm{Bi}-$ chains aligned along the $[1 \overline{1} 0]$ direction with $\mathrm{Bi}-\mathrm{Bi}$ bond length of $3.01 \AA$, which is very close to the sum of the Bi covalent radius ( $2.92 \AA)$. While the ECLS exhibits a well ordered $(1 \times 1)$ surface reconstruction, the InAs missing row model presents a $(1 \times 2)$ surface reconstruction with two inequivalent and strongly buckled $\mathrm{Bi}$-chains along the $[1 \overline{1} 0]$ direction. The $\mathrm{Bi}-$ chains are tilted by $\approx 23^{\circ}$ with respect to the (110) plane, however, the $\mathrm{Bi}-\mathrm{Bi}$ bond lengths (3.07 $\AA$ and $3.03 \AA$ ) are almost the same as those for the ECLS.

In order to verify the energetic stability between these two structural models, we have compared their total energies $(\Delta E)$, which can be written as:

$$
\Delta E=E_{1 \times 2}+E_{I n A s}^{b u l k}-E_{E C L S} .
$$

$E_{1 \times 2}$ and $E_{E C L S}$ represent, respectively, the total energies of the $(1 \times 2)$ model and ECLS. $E_{I n A s}^{\text {bulk }}$ represents the total energy of the InAs bulk, calculated using the same calculational procedure as used for the surface calculations. Our results indicate a small total energy difference: $41 \mathrm{meV} /(1 \times 2)$, in favor of the $(1 \times 2)$ model, $\Delta E=-$ $41 \mathrm{meV} /(1 \times 2)$. Therefore, the $(1 \times 2)$ model is slightly more favourable in energy than the ECLS.

We next have detailed the equilibrium atomic geometry of the ECLS and $(1 \times 2)$ model. For the ECLS, Figs. 1(a) and 1(b), our calculated results are in quite good agreement with the previous $a b$ initio study by Umerski and Srivastava [14]. The topmost Bi-chains are slightly buckled by $0.13 \AA$, where the $\mathrm{Bi}$ adatoms bonded to the substrate In 
(As) are at the "up" ("down") position. Umerski and Srivastava obtained a vertical buckling of $0.15 \AA$ in the same direction. For the III-V (110) clean surfaces the topmost adlayers are tilted considerably due to the cation $\rightarrow$ anion electronic charge transfer, in order to satisfy the electron couting rule (ECR). For instance, for the InAs(110)- $(1 \times 1)$ clean surface, the topmost InAs bonds are buckled by $\approx 0.75 \AA[15]$ with In (As) adatoms in the "down" ("up") positions. The electronic charge transfer occur from In towards As adatoms. As a consequence, the In bonds become almost planar, $s p^{2}-$ like hybridization and an empty $p_{z}$ orbital, while the As bonds become pyramidal: with $s^{2} p^{3}$-like hybridization with a fully occupied dangling bond. Although the Bi-covered ECLS also satisfies the ECR, the electronic charge transfer in the topmost $\mathrm{Bi}$-chains is quite small. The dangling bonds of the $\mathrm{Bi}$ adatoms are fully occupied, since the $\mathrm{Bi}-\mathrm{Bi}$ and $\mathrm{Bi}-\mathrm{As}$ bonds exhibit an electronic charge excess. The $\mathrm{Bi}-\mathrm{In}$ and $\mathrm{Bi}-\mathrm{As}$ bond lengths, see Fig. 1(a), are also in good agreement with the previous theoretical predictions: $2.85 \AA$ ( $\mathrm{Bi}-$ In) and $2.67 \AA$ (Bi-As) [14].

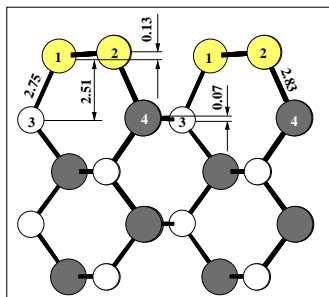

(a)

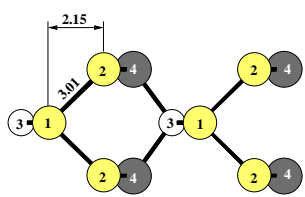

(b)

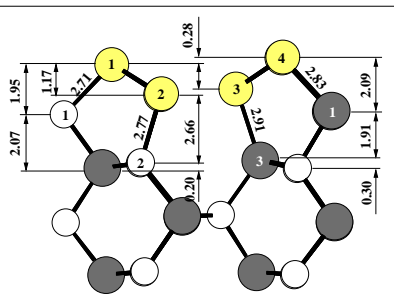

(c)

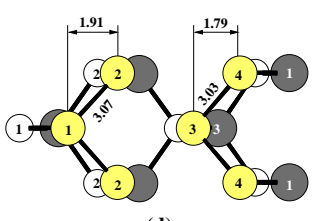

(d)
As

Figure 1. ECLS (a) top and (b) side view, and $(1 \times 2)$ structural model (c) top and (d) side view.

Figures 1(c) and 1(d) exhibit the equilibrium atomic geometry of the $\mathrm{Bi} / \mathrm{InAs}(110)-(1 \times 2)$ surface. The InAs missing row gives rise to two inequivalent $\mathrm{Bi}-$ chains, one bonded to the As atoms and another to the In atoms, both aligned along the $[1 \overline{1} 0]$ direction. The vertical buckling of the $\mathrm{Bi}-$ chains bonded to the As (In) substrate is $1.17 \AA(1.25 \AA)$, which is in good agreement with the experimental results [7]: $1.06 \AA(1.21 \AA)$. The same good agreement has also been verified for the $\mathrm{Bi}-\mathrm{Bi}$ bonds lengths along the chains, $\approx 3.05 \AA$ (exp. [7]: $\approx 2.97 \AA$ ). The Bi-chain bonded to the In substate $\left(\mathrm{Bi}_{3}-\mathrm{Bi}_{4}\right)$ is slightly higher, $0.20 \AA-0.28 \AA$ as compared with the $\mathrm{Bi}$-chain bonded to the As substrate $\left(\mathrm{Bi}_{1}-\mathrm{Bi}_{2}\right)$. This result is in contrast with the experimental findings by Betti and co-workers. They suggested that the $\mathrm{Bi}-$ chain bonded to the As substrate is higher (by $0.46 \AA-$ $0.61 \AA$ ) than the $\mathrm{Bi}-$ chain bonded to the In substrate. Similarly, our calculated $\mathrm{Bi}-\mathrm{In}$ and $\mathrm{Bi}-\mathrm{As}$ bond lengths, see Fig. 1(c), do not agree with the experimental measurements [7]. While we obtained $2.70 \AA$ and $2.83 \AA$ for the $\mathrm{Bi}_{1}-\mathrm{As}_{1}$ and
$\mathrm{Bi}_{4}-\mathrm{In}_{1}$ bond lengths, respectively, the $\mathrm{x}$-ray results [7] indicate: $3.05 \AA\left(\mathrm{Bi}_{1}-\mathrm{As}_{1}\right)$ and $2.62 \AA\left(\mathrm{Bi}_{4}-\mathrm{In}_{1}\right)$. Is is worth to point out that the sum of the covalent radii: $2.67 \AA$ ( $\mathrm{Bi}-$ As) and $2.96 \AA$ (Bi-In), support our calculated results. Further experimental as well as theoretical studies are necessary in order to clarify these experimental/theoretical contradictions.

The total charge distributions along the $\mathrm{Bi}-\mathrm{In}$ and $\mathrm{Bi}-\mathrm{As}$ bonds for the ECLS and $(1 \times 2)$ model are depicted in Figs. 2 and 3, respectively. For the $\mathrm{Bi}-\mathrm{As}$ bonds ( $\mathrm{V}-\mathrm{V}$ bonds), the electronic charge concentration on the As sites is determined by a higher electronegativity of As (2.2) compared with Bi (1.7). Figures 3(a) and 3(b) show the (re)bonding of the $\mathrm{Bi}$ adatoms with the second sublayer, along the InAs missing row: $\mathrm{Bi}_{2}-\mathrm{As}_{2}$ and $\mathrm{Bi}_{3}-\mathrm{In}_{3}$. It is interesting to note that, in general, the total charge densities along the $\mathrm{Bi}-\mathrm{As}$ or Bi-In bonds are almost identical for both structural models, which is in agreement with the similarities of the $\mathrm{Bi}-\mathrm{In}$ $(2.71 \AA-2.77 \AA)$ or $\mathrm{Bi}-\mathrm{As}(2.83 \AA-2.91 \AA)$ equilibrium bond distances.
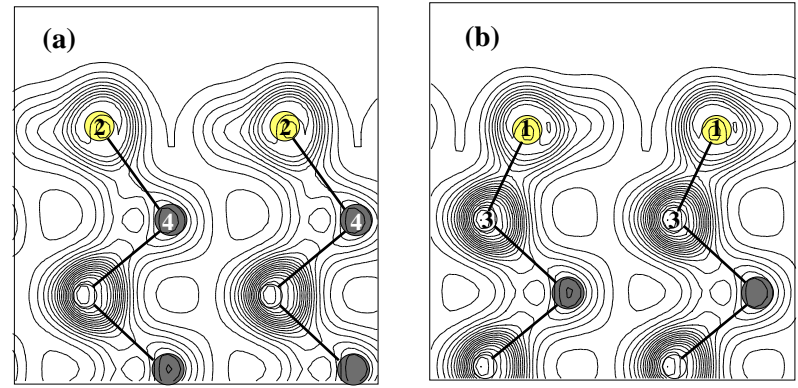

Figure 2. Total charge densities along the (a) Bi-In and (b) Bi-As bonds for the ECLS model.
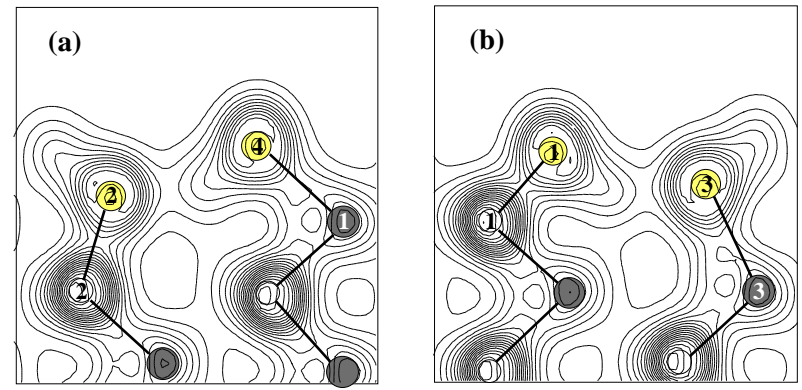

Figure 3. Total charge densities along the (a) Bi-In and (b) Bi-As bonds for the $(1 \times 2)$ model.

\section{Conclusion}

In summary, using first-principles pseudopotential technique, we have investigated the atomic geometry and energetic stability of the $\mathrm{Bi}$-covered InAs(110) surface. We have considered two experimentally proposed structural models: ECLS and $(1 \times 2)$. Our results indicate that the recently proposed $(1 \times 2)$ model [7] is slightly more favourable in energy than the ECLS, thus supporting the experimentally observed ECLS $\rightarrow(1 \times 2)$ structural transition on the Bi/InAs(110) surface. The calculated atomic equilibrium geometry of the 
ECLS compares quite well with the previous works. Similarly for the $(1 \times 2)$ model, our calculated $\mathrm{Bi}-\mathrm{Bi}$ bond lengths agree very well with the experimental measurements. However, the same good agreement has not been verified for the $\mathrm{Bi}-\mathrm{In}$ or $\mathrm{Bi}-\mathrm{As}$ bond lengths, and for the relative vertical positions of the $\mathrm{Bi}-$ chains.

\section{Acknowledgments}

The authors acknowledge financial support from the Brazilian agencies $\mathrm{CNPq}$, and FAPEMIG. This work was partially developed at CENAPAD/CO-MG and CENA$\mathrm{PAD} / \mathrm{SP}$.

\section{References}

[1] C. B. Duke, A. Paton, W. K. Ford, A. Kahn, and J. Carelli, Phys. Rev. B 26, 803 (1982).

[2] P. Mårtenson and R. M. Feenstra, Phys. Rev. B 39, 7744 (1989).

[3] Maria Grazia Betti, Carlo Mariani, N. Jedrecy, R. Pinchaux, A. Ruocco, and M. Sauvage-Simkin, Phys. Rev. B 50, 14336 (1994).

[4] Jun-Hyung Cho, Zhenyu Zhang, Sung-Hoon Lee, and Myung-Ho Kang, Phys. Rev. B 57, 1352 (1998).
[5] R. Ludeke, A. Taleb-Ibrahimi, R. M. Feenstra, and A. B. McLean, J. Vac. Sci. Technol. B 7, 963 (1989).

[6] A. Herrera-Gǿmez, T. Kendelewicz, J. C. Woicik, K. E. Miyano, P. Pianetta, S. Southworth, P. L. Cowan, B. A. Karlin, and W. E. Spicer, J. Vac. Sci. Technol. A 11, 2354 (1993).

[7] Maria Grazia Betti, Davide Berselli, Carlo Mariani, N. Jedrecy, M. Sauvage-Simkin, Y. Garreau, and R. Pinchaux Phys. Rev. B 59, 15760 (1999).

[8] P. Skeath, I. Lindau, C. Y. Su, and W. E. Spicer, J. Vac. Sci. Technol. 19, 556 (1981).

[9] P. Hohenberg, and W. Kohn, Phys. Rev. B 136, B864 (1964).

[10] D. M. Ceperley and B. I. Alder, Phys. Rev. Lett. 45, 566 (1980).

[11] J.P. Perdew and A. Zunger, Phys. Rev. B 23, 5048 (1981).

[12] L. Kleinmann and D.M. Bylander, Phys. Rev. Lett. 48, 1425 (1982);X. Gonze, R. Stumpf and M. Scheffler, Phys. Rev. B 44, 8503 (1991).

[13] G. P. Srivastava, Theoretical Modelling of Semiconductor Surfaces, World Scientific, Singapore, 1999.

[14] A. Umerski and G. P. Srivastava, Phys. Rev. B 51, 2334 (1995).

[15] José Luis Alves, Jörk Hebenstreit, Matthias Scheffler, Phys. Rev. B 44, 6188 (1991). 